\title{
QUIVER CONCOMITANTS ARE OFTEN REFLEXIVE AZUMAYA
}

\author{
LIEVEN LE BRUYN
}

(Communicated by Donald S. Passman)

\begin{abstract}
In this note we show that the concomitants of a quiver with symmetric Ringel form associated to a root from the fundamental chamber is a reflexive Azumaya algebra except for low dimensional anomalities.
\end{abstract}

\section{INTRODUCTION}

Throughout this note we work over an algebraically closed basefield of characteristic zero which we will denote by $\mathbf{C}$. A quiver $Q$ is a fourtuple $\left(Q_{0}, Q_{1}, t, h\right)$ consisting of a finite set of vertices $Q_{0}=\{1, \ldots, n\}$, a set of arrows $Q_{1}$ between these vertices and two maps $T, h: Q_{1} \rightarrow Q_{0}$ assigning to each arrow its tail (resp. its head).

A representation $V$ of the quiver $Q$ consists of a family $\left\{V(i): i \in Q_{0}\right\}$ of finite dimensional $\mathbf{C}$-vectorspaces together with a family of linear maps $\left\{V(\phi): V(t \phi) \rightarrow V(h \phi) ; \phi \in Q_{1}\right\}$. The $n$-tuple $\operatorname{dim}(V(i))_{i} \in \mathbf{N}^{n}$ is called the dimensiontype of the representation $V$. For a fixed dimensiontype $\alpha \in \mathbf{N}^{n}$, the set of all $\alpha$-representations of $Q$ forms a finite dimensional vectorspace

$$
R(Q, \alpha)=\bigoplus_{\phi \in Q_{1}} M_{\phi}(\mathbf{C}),
$$

where $M_{\phi}(\mathbf{C})$ is the $\mathbf{C}$-vectorspace of all $\alpha(h \phi)$ by $\alpha(t \phi)$ matrices with entries in $\mathrm{C}$. We will consider the representation space $R(Q, \alpha)$ as an affine variety with coordinate ring $\mathbf{C}[Q, \alpha]$ and functionfield $\mathbf{C}(Q, \alpha)$.

There is a canonical action of the reductive group $\mathrm{Gl}(\alpha)=\prod_{i=1}^{n} \mathrm{GL}_{\alpha(i)}(\mathbf{C})$ on $R(Q, \alpha)$ by $(g \cdot V)(\phi)=g_{h \phi} V(\phi) g_{t \phi}^{-1}$ for all $g \in \mathrm{GL}(\alpha)$. The $\mathrm{GL}(\alpha)$ orbits in $R(Q, \alpha)$ are precisely the isomorphism classes of representations.

The quotient variety $V(Q, \alpha)=R(Q, \alpha) / \mathrm{GL}(\alpha)$ that is the variety associated to the ring of polynomial invariants $\mathrm{C}[Q, \alpha]^{\mathrm{GL}(\alpha)}$ parametrizes the isomorphism classes of $\alpha$-dimensional semisimple representations. The geometry

Received by the editors December 7, 1989.

1980 Mathematics Subject Classification (1985 Revision). Primary 20G15, 13A20, 14M20, $16 \mathrm{~A} 46$.

Key words and phrases. Invariant theory, representations of quivers, Brauer group.

The author is a research associate of NFWO. 
of this variety has been studied in [LP]. In this paper we are interested in the quiver concomitants, that is we want to investigate the ring of polynomial maps

$$
R(Q, \alpha) \rightarrow M_{l}(\mathbf{C})
$$

which commute with the action of $\operatorname{GL}(\alpha)$ on $R(Q, \alpha)$ as described above and by conjugation on $M_{l}(\mathbf{C})$. Here $l$ denotes $\sum_{i=1}^{n} \alpha(i)$. If $\alpha$ is the dimension vector of a simple representation, then this ring of quiver concomitants which we want to denote by $T[Q, \alpha]$ is a nice affine Noetherian prime p.i.-ring. In analogy with trace rings of generic matrices [L2] one can ask whether it is a maximal order, or even stronger, a reflexive Azumaya algebra. Although we believe that this will be virtually always the case (discarting possible low dimensional counterexamples as for trace rings the case of two generic 2 by 2 matrices) we will restrict our attention here to the special case of quivers with a symmetric Ringel form.

We note that this special case is of interest even for finite dimensional algebras. For, the preprojective algebra of a wild hereditary basic algebra is known to be a quotient of the path algebra of a quiver with symmetric Ringel form. In a forthcoming paper we will apply the results obtained here to the study of finite dimensional representations of these preprojective algebras.

\section{THE FUNDAMENTAL CHAMBER AND SIMPLE REPRESENTATIONS}

The dimension vectors of simple representations were described in [LP]. Let us briefly recall the result here. If $Q$ is an arbitrary quiver, we have the bilinear Ringel form $R$ on $\mathrm{Z}^{n}$ determined by $R\left(e_{i}, e_{j}\right)=\delta_{i j}-r_{i j}$ where $e_{i}=\left(\delta_{i j}\right)_{j}$ is the standard basis for $\mathbf{Z}^{n}$ and $r_{i j}$ is the number of oriented arrows from $i$ to $j$. Further, we call a full subquiver $Q^{\prime}$ of $Q$ a club provided every couple of its vertices belongs to an oriented cycle within $Q^{\prime}$. Then: $\alpha \in \mathbf{N}^{n}$ is the dimension vector of a simple representation iff either $\operatorname{supp}(\alpha)$ is one oriented cycle and all $\alpha(i)$ are equal to 1 or $\operatorname{supp}(\alpha)$ is a noncycle club and for all $i$ we have $R\left(e_{i}, \alpha\right) \leq 0$ and $R\left(e_{i}, \alpha\right) \leq 0$.

Further, we recall that the definition of the Tits form associated to the quiver $Q$ is determined by $T(\alpha, \beta)=\frac{1}{2}(R(\alpha, \beta)+R(\beta, \alpha))$. The fundamental chamber, see for example [Ka], is then defined to be the set $\mathscr{F}(Q)$ consisting of those nonzero vectors in $\mathbf{N}^{n}$ such that $T\left(e_{i}, \alpha\right) \leq 0$ for all $i$ and $\operatorname{supp}(\alpha)$ is a connected graph.

Combining these facts we have the following

Lemma 1. For any quiver $Q$ with symmetric Ringel form the set of dimension vectors of simple representations coincides with the fundamental chamber $\mathscr{F}(Q)$.

Therefore, the set of dimension vectors of simple representations is a solid cone in $\mathbf{N}^{n}$ with bounding hyperplanes determined by the linear equations 
$R\left(\alpha, e_{i}\right)=0$. There is just one counterexample: the two point, two edges quiver.

\section{A COMBINATORIAL LEMMA}

In this section we single out an easy but boring combinatorial lemma which will be essential in the proof.

Lemma 2. If $\alpha, \beta, \gamma$ are dimension vectors of simple representations of a quiver $Q$ with symmetric Ringel form such that $\alpha=\beta+\gamma$ and $\alpha(i) \geq 2$ for all $i$, then $R(\beta, \gamma)<-1$ or we are in one of the following three situations:

(A) $\alpha=(2,2,2), \beta=\gamma=(1,1,1)$ and $Q$ is the quiver

(B) $\alpha=(2,2), \beta=\gamma=(1,1)$ and $Q$ is the quiver

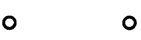

(C) $\alpha=2, \beta=\gamma=1$ and $Q$ is the two loop quiver.

Proof. Let us assume that $R(\beta, \gamma)=R(\gamma, \beta) \geq-1$. Then, since $R\left(\beta, e_{i}\right) \leq 0$ we know that for all but at most one $i \in \operatorname{supp}(\gamma)$ (say $\left.i_{0}\right)$ we have $R\left(\beta, e_{i}\right)=0$ and $R\left(\beta, e_{i_{0}}\right)=-1, \gamma\left(i_{0}\right)=1$. Similarly, for all but at most one $j \in \operatorname{supp}(\beta)$ (say $\left.j_{0}\right)$ we have $R\left(\gamma, e_{j}\right)=0$ and $R\left(\gamma, e_{j_{0}}\right)=-1, \beta\left(j_{0}\right)=1$.

Next, we claim that $\operatorname{supp}(\alpha)=\operatorname{supp}(\beta)=\operatorname{supp}(\gamma)$. For, suppose $i \in$ $\operatorname{supp}(\gamma)-\operatorname{supp}(\beta)$ then $i \neq i_{0}$ since then $\alpha\left(i_{0}\right)=\gamma\left(i_{0}\right)=1$. So, $R\left(\beta, e_{i}\right)=0$ but then $R\left(e_{j}, e_{i}\right)=0$ for all $j \in \operatorname{supp}(\beta)$. This would imply that the matrix defining the Ringel form has the following shape

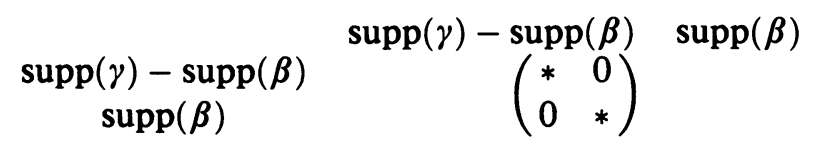

which is impossible by the clubassumption on $\operatorname{supp}(\alpha)$. Hence $\operatorname{supp}(\gamma) \subset$ $\operatorname{supp}(\gamma)$ and by $\operatorname{symmetry}$ we obtain $\operatorname{supp}(\gamma)=\operatorname{supp}(\beta)$ as claimed.

Consider first the case when $i_{0} \neq j_{0}$, then

$$
R\left(\gamma, i_{0}\right)=R\left(e_{i_{0}}, e_{i_{0}}\right)+\sum_{j \neq i_{0}} \gamma(j) R\left(e_{j}, e_{i_{0}}\right)=0 .
$$

By an argument as above not all $R\left(e_{j}, e_{i_{0}}\right)$ can be zero. So, $R\left(e_{i_{0}}, e_{i_{0}}\right)=1$ (i.e. there is no loop in $i_{0}$ ) and there is a unique $j$ (say $\left.i_{1}\right)$ such that $R\left(e_{i_{1}}, e_{i_{0}}\right) \neq 0$ 
and $R\left(e_{i_{1}}, e_{i_{0}}\right)=-1, \gamma\left(i_{1}\right)=1$. If $i_{1} \neq j_{0}$ then

$$
\begin{aligned}
0 & =R\left(\gamma, e_{i_{1}}\right)=R\left(e_{i_{1}}, e_{i_{1}}\right)-1+\sum_{k \neq i_{0}, i_{1}} \gamma(k) R\left(e_{k}, e_{i_{1}}\right) \\
& \leq \sum_{k \neq i_{0}, i_{1}} \gamma(k) R\left(e_{k}, e_{i_{1}}\right)
\end{aligned}
$$

whence for all $k \neq i_{0}, i_{1}$ we get $R\left(e_{k}, e_{i_{1}}\right)=0$. Similarly, if $i_{1}=j_{0}$ we can repeat the same argument with $\beta$ instead of $\gamma$. Therefore, the quiver $Q$ must be

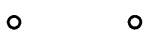

and $\gamma=(1,1)$ contradicting that $\alpha$ is the dimension vector of a simple representation.

So, we are reduced to the case that $i_{0}=j_{0}=i$. That is we have $\beta(i)=\gamma(i)=$ $1, R\left(\beta, e_{i}\right)=R\left(\gamma, e_{i}\right)=-1$ and for all $k \neq i$ we get $R\left(\beta, e_{k}\right)=R\left(\gamma, e_{k}\right)=0$. Now,

$$
-1=R\left(\beta, e_{i}\right)=\beta(i) R\left(e_{i}, e_{i}\right)+\sum_{k \neq i} \beta(k) R\left(e_{k}, e_{i}\right) .
$$

Assume first that there are no loops in $i$, i.e. $R\left(e_{i}, e_{i}\right)=1$, then there are three possible situations:

(I) For all $k \neq i, l, m$ we have $R\left(e_{k}, e_{i}\right)=0$ and $R\left(e_{l}, e_{i}\right)=R\left(e_{m}, e_{i}\right)=$ $-1, \beta(l)=\beta(m)=1$. Then similarly also $\gamma(l)=\gamma(m)=1$. But then $0=R\left(\beta, e_{l}\right)=\beta(l) R\left(e_{l}, e_{l}\right)+\beta(i) R\left(e_{l}, e_{i}\right)+\sum_{k \neq i, l} \beta(k) R\left(e_{k}, e_{l}\right)$ which is smaller or equal to the last term which must be negative unless for all $k \neq i, l$ we have $R\left(e_{k}, e_{l}\right)=0$. Similarly we have for all $k \neq i, m$ that $R\left(e_{k}, e_{m}\right)=0$. This gives our first counterexample: the quiver $Q$ is then

and $\alpha=(2,2,2), \beta=\gamma=(1,1,1)$

(II) For all $k \neq i, l$ we have $R\left(e_{k}, e_{i}\right)=0$ and $R\left(e_{l}, e_{i}\right)=-1, \beta(l)=2$ (and similarly $\gamma(l)=2)$. But then $0=R\left(\beta, e_{l}\right)$ is smaller or equal than $1+\sum_{k \neq i, l} \beta(k) R\left(e_{k}, e_{l}\right)$. Hence for all $k \neq i, l, m$ we must have $R\left(e_{k}, e_{l}\right)=0$ and $R\left(e_{m}, e_{l}\right)=-1, \beta(m)=1$. But then $R\left(\beta, e_{m}\right)$ is smaller or equal than -1 which is a contradiction, i.e. this case does not occur.

(III) For all $k \neq i, l$ we have $R\left(e_{k}, e_{i}\right)=0$ and $R\left(e_{l}, e_{i}\right)=-2, \beta(l)=1$ (and similarly $\gamma(l)=1$ ). But then $R\left(\beta, e_{l}\right)$ is smaller or equal than -1 so this case too cannot occur.

Next assume there is a single loop in $i$, i.e. $R\left(e_{i}, e_{i}\right)=0$, then for all $k \neq i, l$ we have that $R\left(e_{k}, e_{i}\right)=0$ and $R\left(e_{l}, e_{i}\right)=-1, \beta(l)=1$ and 
similarly $\gamma(l)=1$. But then we obtain from $R\left(\beta, e_{l}\right)=0$ that there are no loops at $l$ and for all $k \neq i, l$ we have $R\left(e_{k}, e_{l}\right)=0$. Hence, we get our second counterexample: the quiver $Q$ is

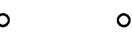

and $\alpha=(2,2), \beta=\gamma=(1,1)$.

The only remaining case gives our third counterexample: the two loop quiver in $i$ and $\alpha=2, \beta=\gamma=1$.

\section{THE SINGULAR LOCUS OF THE QUOTIENT VARIETY}

$\tau=\left(m_{1}, \alpha_{1} ; \cdots ; m_{k}, \alpha_{k}\right)$ is said to be an admissible $\alpha$-representation type if each of the $\alpha_{i}$ are dimension vectors of simple representations of the quiver $Q$ and $\alpha=\sum_{i=1}^{k} m_{i} \alpha_{i}$. With $V(\tau)$ we denote the set of points in the quotient variety $V(Q, \alpha)=R(Q, \alpha) / \mathrm{GL}(\alpha)$ corresponding to semisimple representations of type $\tau$. These sets form a finite stratification into locally closed smooth algebraic subvarieties of $V(Q, \alpha)$, see [LP]. The set of points corresponding to simple representations forms an open subvariety $V(1, \alpha)$ of $V(Q, \alpha)$.

Proposition 1. Let $\alpha$ be a root from the fundamental chamber of the quiver $Q$ with symmetric Ringel form such that $\alpha(i) \geq 2$ for all vertices $i$, then the set of smooth points of the quotient variety $V(Q, \alpha)$ coincides with $V(1, \alpha)$ except for the cases (A), (B) and (C) mentioned above. In those cases the quotient variety is the five dimensional affine space.

Proof. Let us first consider the general case. If $\tau \neq(1, \alpha)$ it follows from the stratification result of [LP] that $V(\tau)$ lies in the closure of one of the subvarieties $V(1, \beta ; 1, \gamma)$ where $\beta$ and $\gamma$ are dimension vectors of simple representations (use the fact that in this case the set of all simple dimensionvectors is a convex cone). It is clear that $V(1, \alpha)$ contains only smooth points. Assume now that there are more smooth points. Since they form an open subvariety there must be smooth points in at least one of the $V(1, \beta ; 1, \gamma)$. Let us compute the étale slice representation in such a point, see [LP] for more details. The coordinate ring of the slice representation is the ring of polynomial invariants of the quiver $Q(\beta, \gamma)$ on two points with $1-R(\beta, \beta)$ loops in vertix $1,1-R(\gamma, \gamma)$ loops in vertex 2 and there are $-R(\beta, \gamma)$ directed arrows from 1 to 2 and from 2 to 1 ; the corresponding dimensionvector is $(1,1)$. Since this is a torus action one can readily verify that the coordinatering is a polynomial ring in $2-R(\beta, \beta)-R(\gamma, \gamma)$ indeterminates over the coordinate ring of rank one $-R(\beta, \gamma)$ by $-R(\beta, \gamma)$ matrices. This last variety always has a singularity in the origin provided that $R(\beta, \gamma)<-1$ which is the case by Lemma 2 . Therefore none of the varieties $V(1, \beta ; 1, \gamma)$ can contain smooth points finishing the proof. 
As for the special cases: we know that the coordinatering of the quotient variety is generated by traces of oriented cycles in the quiver. In case (A) this ring is generated by $\operatorname{Tr}\left(A_{21}, A_{12}\right), \operatorname{Tr}\left(A_{23}, A_{32}\right), \operatorname{Tr}\left(\left(A_{21} A_{12}\right)^{2}\right), \operatorname{Tr}\left(\left(A_{23}, A_{32}\right)^{2}\right)$ and $\operatorname{Tr}\left(A_{21} A_{12} A_{23} A_{32}\right)$. In case (B) it is generated by $\operatorname{Tr}\left(A_{1}\right), \operatorname{Tr}\left(A_{1}^{2}\right), \operatorname{Tr}\left(A_{12} A_{21}\right)$, $\operatorname{Tr}\left(\left(A_{12} A_{21}\right)^{2}\right)$ and $\operatorname{Tr}\left(A_{1} A_{12} A_{21}\right)$. In case (C) it is generated by $\operatorname{Tr}\left(A_{1}\right)$, $\operatorname{Tr}\left(B_{1}\right), \operatorname{Tr}\left(A_{1}^{2}\right), \operatorname{Tr}\left(B_{1}^{2}\right)$ and $\operatorname{Tr}\left(A_{1} B_{1}\right)$.

\section{Concomitants are reflexive AzUmaya algebras}

Let us start by recalling the definition of a reflexive Azumaya algebra, see for example [Yu or L]. Let $\Gamma$ be an order over a normal domain $R$ in a central simple algebra $\Sigma$ over its field of fractions $K$. Then $\Lambda$ is said to be reflexive Azumaya iff the localization at each height one prime ideal $p$ of $R, \Lambda_{p}$ is an Azumaya algebra over $R_{p}$ and if $\Lambda$ is a reflexive $R$-module. Note that a reflexive Azumaya algebra is a maximal order.

Proposition 2. Let $\alpha$ be a root from the fundamental chamber of a quiver $Q$ with symmetric Ringel form such that $\alpha(i) \geq 2$ for every vertex $i$ then the ring of quiver concomitants $T(Q, \alpha)$ is a reflexive Azumaya algebra except for the three cases mentioned above where it is a maximal order.

Proof. In view of the Artin-Procesi result (see, for example, [AR]), this result comes down to checking that the codimension of the closed subvariety corresponding to decomposable semisimple $\alpha$-representations is greater than or equal to two. Note that since $\alpha$ is the dimension vector of a simple representation it follows from [LP] that the dimension of the quotient variety $V(Q, \alpha)$ is equal to $1-R(\alpha, \alpha)$. Moreover, it follows from the stratification result mentioned above that every decomposable semisimple representation lies in the closure of one of the (locally closed) subvarieties $V(1, \beta ; 1, \gamma)$ which are of dimension $2-R(\beta, \beta)-R(\gamma, \gamma)$. Since $\alpha=\beta+\gamma$ and the Ringel form is assumed to be symmetric, the condition that the codimension of $V(1, \beta ; 1, \gamma)$ is at least two comes down to the condition $R(\beta, \gamma)<-1$ which is the content of Lemma 2 . This finishes the proof in the general case.

In the special cases $(\mathrm{A}),(\mathrm{B})$ and $(\mathrm{C})$ the quiver concomitants are easily seen to be isomorphic to $M_{3}\left(\mathbf{T}_{2,2}\right)$, resp. $M_{2}\left(\mathbf{T}_{2,2}\right)$ and $\mathbf{T}_{2,2}$ where $\mathbf{T}_{2,2}$ is the trace ring of two generic two by two matrices which is known to be a (ramified) maximal order.

Reflexivity follows as in the proof of the Artin-Schofield [L2] result from the fact that there is no blowing up in the extension $\mathbf{C}[Q, \alpha]^{\mathrm{GL}(\alpha)} \subset \mathbf{C}[Q, \alpha]$ in view of the foregoing results and the Luna slice theorem.

In contrast to trace rings of generic matrices it is no longer true that the ring of polynomial invariants is a unique factorization domain and consequently that the ring of quiver concomitants has trivial centralizing or normalizing classgroup. An easy but interesting example is given in [LP1]: the quiver concomitants of the two point quiver with two directed arrows from 1 to 2 and from 
2 to 1 associated with the dimension vector $(1,1)$ gives a reflexive Azumaya maximal order which even has global dimension three but it has a height one prime which is not generated by a normalizing element since the classgroup of the ring of polynomial invariants is isomorphic to $\mathbf{Z}$.

Finally we note that the cohomological interpretation of the reflexive Brauer group obtained in [L] is explained in these cases by Proposition 1.

\section{REFERENCES}

[Ar] M. Artin, On Azumaya algebras and finite dimensional representations of rings, J. Algebra 11 (1969), 532-563.

[Ka] V. Kac, Infinite root systems, representations of graphs and invariant theory, Invent. Math. 56 (1980), 57-92.

[L] L. Le Bruyn, A cohomological interpretation of the reflexive Brauer group, J. Algebra 105 (1987), 250-254.

[L2] _ The Artin-Schofield theorem and some consequences, Comm. Algebra 14 (8) (1986), 1439-1455.

[LP] L. Le Bruyn and C. Procesi, Semisimple representations of quivers, Trans. Amer. Math Soc. (to appear).

[LP1] _ Etale local structure of matrixinvariants and concomitants (Proc. Algebraic Groups Utrecht, 1986), Lecture Notes in Math., vol. 1271, Springer-Verlag 1987, pp. 143-176.

[Yu] S. Yuan, Modules and algebra classgroups over Noetherian integrally closed domains, J. Algebra 32 (1974), 405-417.

DePARTMENT OF MATHEMATICS, UNIVERSitAire INSTELling ANTWERPEN, UNIVERSITEITSPLEIN 1, B-2610 ANTWERPEN, BELGIUM 